\title{
Cardioplastia esôfago diafragmática em filhote de cão com megaesôfago congênito
}

\author{
[Diaphragmatic esophageal cardioplasty in a puppy with congenital megaesophagus]
}

\section{"Relato de caso/Case report"}

\author{
Victor Manuel de Lacerda Freitas ${ }^{1 *}$, Gessica dos Santos Araújo ${ }^{2}$, Reginaldo Pereira de Sousa Filho ${ }^{3}$, \\ Aline Silveira Feitosa ${ }^{4}$, Keytyanne de Oliveira Sampaio ${ }^{5}$, Tainara Micaele Bezerra Peixoto ${ }^{6}$ \\ ${ }^{1}$ Universidade Federal de Campina Grande, Patos, Paraíba, Brasil. \\ ${ }^{2}$ Programa de Pós-Graduação em Ciências Veterinárias, Universidade Estadual do Ceará, Fortaleza, Ceará, Brasil. \\ ${ }^{3}$ Médico Veterinário da Empresa Catus Medicina Felina, Fortaleza, Ceará, Brasil. \\ ${ }^{4}$ Faculdade Terra Nordeste, Caucáia, Ceará, Brasil. \\ ${ }^{5}$ Departarmento de Medicina Veterinária, Universidade Federal Rural de Pernambuco, Recife, Pernambuco, Brasil. \\ ${ }^{6}$ Programa de Pós-Graduação em Ciência Animal,Universidade Estadual Norte Fluminense Darcy Ribeiro, Campos dos \\ Goitacazes, Rio de Janeiro, Brasil. \\ *Autor para correspondência/Corresponding author: E-mail: victorlacerdafreitas @gmail.com
}

\section{Resumo}

O megaesôfago é uma dilatação esofágica, resultante de uma doença primária ou secundária, determinando distúrbio motor da deglutição. Dos vários procedimentos terapêuticos preconizados, o tratamento cirúrgico representa uma opção, por proporcionar alívio dos sinais clínicos e melhorar o estado nutricional. Entretanto, contestações surgem quanto à melhor técnica cirúrgica em animais com megaesôfago após tratamento conservador prévio. O presente trabalho teve como objetivo descrever a técnica cirúrgica denominada cardioplastia esôfago diafragmática em filhote de cão com megaesôfago congênito generalizado. Nesse relato, um filhote canino foi levado ao atendimento médico veterinário devido a regurgitações cada vez mais frequentes. Na radiografia contrastada, foi observado que o esôfago torácico se encontrava acentuadamente dilatado, sendo a imagem sugestiva de megaesôfago generalizado. Devido ao agravo dos episódios de regurgitação e perda de peso progressiva após duas semanas de tratamento dietético conservador, foi decidido à realização da cirurgia pela técnica de cardioplastia esôfago diafragmático. Essa técnica consiste em reduzir artificialmente a paralisia da porção torácica final do esôfago e da cardia, através da atividade contrátil do diafragma durante os ciclos respiratórios. No primeiro retorno, o tutor relatou que a cadela ainda regurgitava esporadicamente, mas com uma diminuição significante da frequência que os mesmos ocorriam.

Palavras-chave: diafragma; regurgitação; cirurgia.

\begin{abstract}
Megaesophagus is an esophageal dilatation resulting from a primary or secondary disease, causing a motor swallowing disorder. Of the various therapeutic procedures recommended, surgical treatment is an option, since it provides relief of clinical signs and improves nutritional status. However, challenges arise regarding the best surgical technique in animals with megaesophagus after previous conservative treatment. The present study aimed to describe the surgical technique called diaphragmatic esophageal cardioplasty in a dog with generalized congenital megaesophagus. In this report, a puppy was taken to the veterinary medical service due to increasingly frequent regurgitations. On contrast radiography, it was observed that the thoracic esophagus was markedly dilated, the image suggestive of generalized megaesophagus. Due to worsening of episodes of regurgitation and progressive weight loss after two weeks of conservative dietary treatment, it was decided to perform a diaphragmatic esophageal cardioplasty surgery. This technique consists in artificially reducing the paralysis of the final thoracic portion of the esophagus and the cardia through the contractile activity of the diaphragm during respiratory cycles. On the first follow-up, the owner reported that the bitch still regurgitated sporadically, but with a significant decrease in frequency.
\end{abstract}

Keywords: diaphragm; regurgitation; surgery. 


\section{Introdução}

O megaesôfago é uma doença caracterizada pela diminuição ou deficiência dos plexos nervosos intramurais do esôfago, constituindo distúrbio de motilidade esofágico ao ato de ingestão (Celano et al., 2007). Podendo ser classificado em congênito, idiopático ou secundário adquirido (Washabau, 2004). Quando este se apresenta na forma congênita ocorre uma progressiva desordem da atividade motora e destruição de 50 a $95 \%$ das células nervosas, trazendo como consequência a flacidez parcial ou total do esôfago, suscitando acúmulo de alimento no mesmo. (Longshore, 2008). A regurgitação e o subdesenvolvimento de filhotes após o desmame pode indicar um diagnóstico de megaesôfago congênito devido à hipomotilidade e à dilatação generalizada (Longshore, 2008; Tanaka et al., 2010).

Ao exame físico, auscultação de líquidos e alimentos retidos no esôfago, halitose e dor associada à palpação da região do esôfago são achados comuns (Radlinsk, 2015). Sinais respiratórios como corrimento nasal mucopurulento, crepitações respiratórias, taquipneia e dispneia devido a pneumonia por aspiração também são encontrados com frequência (Tanaka et al., 2010). Os métodos de diagnóstico definitivo disponíveis na medicina veterinária incluem a radiografia simples, a radiografia contrastada e a endoscopia (Washabau, 2004). Segundo Willard (2015), a radiografia contrastada é indicada quando a radiografia simples não consegue fechar o diagnóstico, sendo o sulfato de bário ou o contraste contendo iodo os mais utilizados. A associação do exame clínico e de imagem é suficiente para a chegada ao diagnóstico (Willard, 2015).

Classicamente, o megaesôfago é tratado através de mudanças na dieta, seguindo um padrão dietético conservador, a fim de evitar o agravamento da dilatação e da aspiração (Willard, 2015). Entretanto, diante do insucesso desse tratamento tem-se sugerido a realização de técnicas cirúrgicas utilizadas em seres humanos adaptadas para a medicina veterinária (Tanaka et al., 2010). Técnicas cirúrgicas, como a esofagomiotomia de Heller modificada e cardioplastia de Wendel são descritas para o tratamento dessa afecção, mas sem sucesso devido às complicações de refluxo gástrico e esofagites, além de serem técnicas laboriosas e com alto risco operatório (Torres, 2000). Contudo, outra técnica aparentemente promissora, a cardioplastia esôfago diafragmática, foi descrita por Torres (2000) com uma taxa de sucesso significativamente relevante em cães. A técnica consiste na utilização da contração do musculo diafragmático durante o ciclo respiratório, permitindo a entrada de alimento para estômago. Assim, o presente trabalho teve como objetivo avaliar e descrever a técnica cirúrgica de cardioplastia esôfago diafragmática em filhote de cão com megaesôfago congênito generalizado.

\section{Descrição do Caso}

Foi atendido no Hospital Veterinário Professor Sylvio Barbosa Cardoso (HVSBC), Fortaleza-CE, uma Pinscher, oito meses de idade, pesando $2,0 \mathrm{~kg}$. O animal foi levado ao atendimento médico veterinário devido à observação, pelos tutores, de "vômitos" que estavam aumentando a frequência e que aconteciam logo após a alimentação desde os quatro meses de idade. As mucosas estavam normocoradas, tempo de preenchimento capilar normal, linfonodos à palpação apresentaram-se normais, assim como sua temperatura. À auscultação cardíaca não apresentou nenhuma alteração, entretanto, na auscultação pulmonar foram identificados estertores no campo pulmonar do hemitórax direito. Suspeitando-se que o animal apresentava megaesôfago, realizou-se exames complementares, incluindo hemograma completo, bioquímica sérica, aferição da pressão arterial, ecodopplercardiograma, radiografia simples e contrastada do tórax (esofagograma) nas incidências latero-lateral e ventro-dorsal.

No hemograma, as alterações observadas foram uma leve eritropenia e trombocitopenia. Não foi identificada nenhuma alteração nos valores da pressão arterial ou no ecodopplercardiograma. $\mathrm{Na}$ radiografia contrastada foi observado uma dilatação do esôfago torácico (Figura 1). Na radiografia simples, um deslocamento ventral da traqueia foi identificado e um padrão alveolar estava presente na periferia do lobo médio direito do pulmão. Posteriormente ao exame clínico e aos resultados dos exames complementares, a suspeita de megaesôfago congênito foi confirmada. Portanto, foi recomendado aos tutores o tratamento dietético conservador e, caso o paciente não apresentasse melhora, seria realizado o procedimento cirúrgico denominado cardioplastia esôfago diafragmática.

Para o tratamento da pneumonia por aspiração foi prescrito amoxicilina com 
clavulanato de potássio $(20 \mathrm{mg} / \mathrm{kg}, \mathrm{PO}$, b.i.d.) durante 14 dias. Foi recomendado que o animal se alimentasse em estação, com o apoio dos membros pélvicos, com alimento pastoso oferecido cinco vezes ao dia em pequenos volumes de acordo com a necessidade energética diária de manutenção. A paciente ainda deveria permanecer em estação por mais 10 minutos após terminar de se alimentar. Duas semanas depois, o paciente foi levado para consulta de retorno. O tutor relatou que o animal continuava regurgitando frequentemente e estava perdendo peso, sendo então encaminhado para cirurgia.

A paciente foi levada para a sala préoperatória, onde foi administrada a medicação préanestésica: metadona $(0,3 \mathrm{mg} / \mathrm{kg}, \quad \mathrm{IM}) \mathrm{e}$ acepromazina $(0,02 \mathrm{mg} / \mathrm{kg}, \mathrm{IM})$. Após a medicação pré-anestésica, foi realizado a tricotomia em todo o hemitórax esquerdo. Em seguida, o animal foi levado para a sala cirúrgica, na qual o referido foi pré-oxigenado por 5 minutos e, então, feita a indução anestésica com quetamina $(1 \mathrm{mg} / \mathrm{kg}, \mathrm{IV})$, midazolan $(0,2 \mathrm{mg} / \mathrm{kg}$, IV), citrato de fentanila $(2,5 \mu \mathrm{g} / \mathrm{kg}, \mathrm{IV})$, lidocaína ( $2 \mathrm{mg} / \mathrm{kg}, \mathrm{IV})$ e propofol $(3 \mathrm{mg} / \mathrm{kg}, \mathrm{IV})$. Em seguida, a cadela foi entubada com sonda traqueal de número 4 e a anestesia foi mantida com isoflurano vaporizado com oxigênio $100 \%$ no sistema aberto com baraka. Durante todo o procedimento, foi realizado monitoramento cardíaco com o eletrocardiograma. Também foram utilizados capnógrafo e oximetria de dedo e os dados referentes à pressão arterial e à temperatura estavam disponíveis em tempo real. No transcirúrgico foi aplicado citrato de fentanila $(5 \mu \mathrm{g} / \mathrm{kg}, \mathrm{IV})$, para auxílio analgésico, e cefalotina $(30 \mathrm{mg} / \mathrm{kg}, \mathrm{IV})$, como antibiótico de escolha.

Posicionou-se o paciente em decúbito lateral direito, sendo realizada a incisão ao longo do oitavo espaço intercostal esquerdo. Após a toraco-

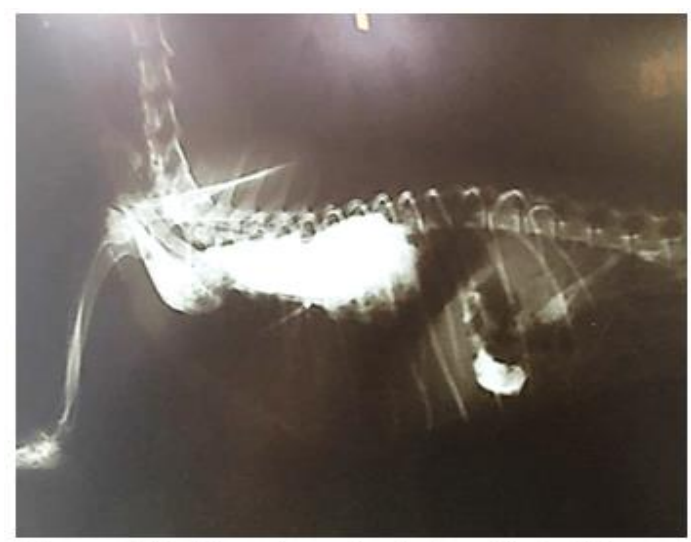

Figura 1. Radiografia contrastada projeção láterolateral onde foi evidenciado o esôfago com aumento generalizado. tomia, as principais estruturas foram identificadas: artéria aorta, nervo vago, esôfago e diafragma (Figura 2). Dissecou-se a pleura mediastínica e com o auxílio de uma gaze úmida o esôfago foi tracionado cranialmente. Em seguida, incidiu-se na metade esquerda do ligamento freno-esofágico, liberando o esôfago de suas aderências na região do hiato. Foi efetuada uma incisão de três centímetros aproximadamente, dorsal e ventral ao esôfago, no diafragma. Finalmente, foi realizada a ressecção lateral em formato semilunar do tecido diafragmático partindo das incisões previamente realizadas (Figura 3). O diafragma foi suturado a parede do esôfago com fio nylon 3-0 no padrão simples interrompido, refazendo a região do hiato (Figura 4). Então, foi colocado o dreno torácico no sétimo espaço intercostal para drenagem a cada quatro horas ou dependendo da quantidade de efusão produzida, sendo removido com 24 horas. $\mathrm{O}$ tórax foi fechado, utilizando-se fio nylon 2-0 ao redor das costelas, sendo utilizado o padrão simples interrompido. As camadas musculares foram fechadas individualmente com poliglactina 910 $\left(\right.$ Vicryl $\left.{ }^{\mathrm{TM}}\right)$, usando padrão simples continuo e pele com nylon 3-0, em padrão simples interrompido. Prescreveu-se cefalexina $(30 \mathrm{mg} / \mathrm{kg}, \mathrm{PO}$, b.i.d.) durante 20 dias, meloxicam $(0,1 \mathrm{mg} / \mathrm{kg}, \mathrm{PO}$, s.i.d.) dias e tramadol $(2,0 \mathrm{mg} / \mathrm{kg}$, PO, t.i.d. $)$ por um período de 5 dias. Foi recomendado que o animal ficasse internado para observação por pelo menos 24 horas, repouso, restrição de espaço, alimentação pastosa com pequenos volumes várias vezes ao dia e em estação. Dez dias após a realização do procedimento o animal apresentava-se clinicamente bem, além de normoréxico, normodipsia e com episódios de regurgitação menos frequentes. Quinze dias após a realização do procedimento, o paciente veio a óbito subitamente, sendo a necropsia não autorizada pelo tutor.

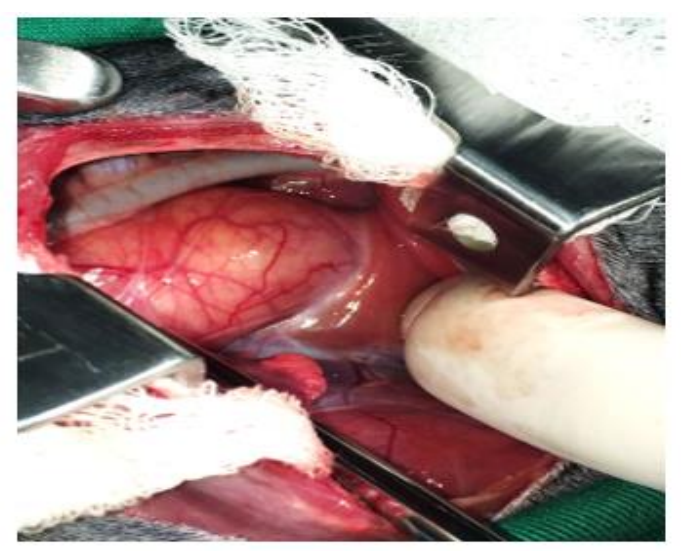

Figura 2. Visualização das principais estruturas intratorácicas após a abertura do tórax no oitavo espaço intercostal esquerdo. 


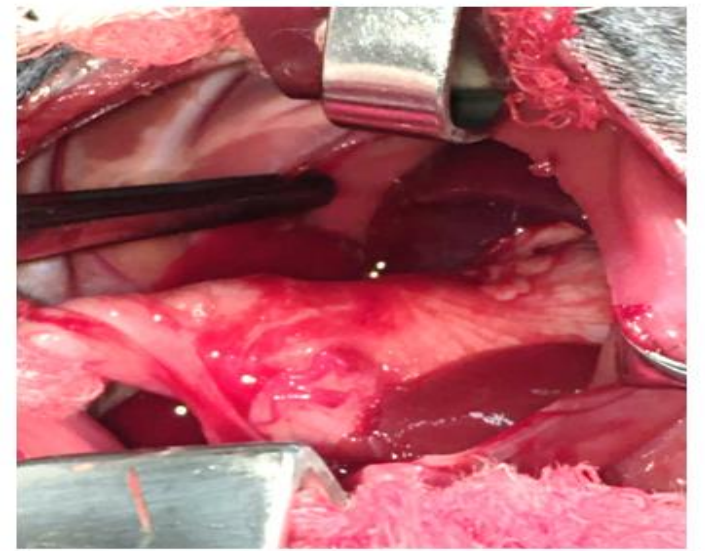

Figura 3. Após remoção de parte do tecido diafragmático ao redor da metade esquerda do esôfago.

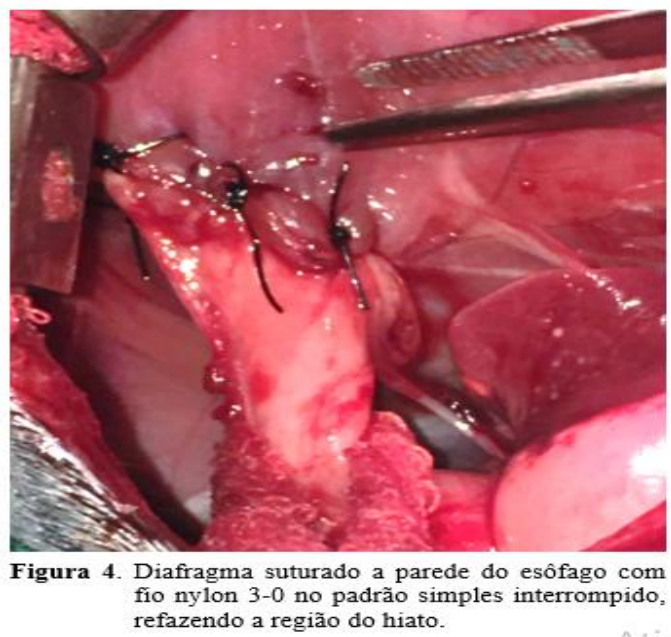

após a desmama, quando a troca da alimentação liquida para solida. Entretanto, existem relatos de apresentação dos sinais clínicos de forma mais tardia, apesar de não ser mais comum, com cinco meses de idade (Alves, 2013). Portanto, o paciente do caso em questão foi diagnosticado com megaesôfago congênito, pois não foi diagnosticada nenhuma patologia primaria para o desenvolvimento de megaesôfago e a forma idiopática, que é a mais comum em cães adultos, foi descartada já que o mesmo era um filhote de seis meses quando os tutores observaram os primeiros sinais clínicos.

A ocorrência de regurgitação de alimentos ocorre logo após sua ingestão (Radlinsk, 2015). Por isso, ressalta-se o perigo causado pela pneumonia por aspiração e a importância da realização do manejo dietético conservador (Langshore, 2008). Sinais clínicos relacionados ao sistema respiratório podem ser os únicos relatos do proprietário (Radlinsk, 2015), entretanto, não foi relatada, pelo tutor, nenhuma alteração respiratória. O diagnóstico é baseado nos sinais clínicos e nos exames radiográficos, em sua maioria, contrastados com sulfato de bário para uma melhor visualização do esôfago dilatado (Willard, 2015). As radiografias torácicas indicam complicações da doença esofágica, incluindo a pneumonia por aspiração, a efusão pleural, a mediastinite e o pneumotórax (Washabau, 2004). Nas radiografias simples cervicais e torácicas é visto uma dilatação esofágica , com acúmulo de gás, fluido ou ingesta, e a traqueia pode estar deslocada ventralmente (Sturgess e Dunn, 2001; Langshore, 2008), como visto no presente relato onde foi observado uma dilatação generalizada do esôfago torácico de modo que a traqueia e o coração estavam deslocados ventralmente, além de um padrão 
alveolar gerado por uma possível pneumonia por aspiração presente na periferia do lobo médio direito do pulmão.

Além dos exames radiográficos, podem ser solicitados exames hematológicos e bioquímicos, que segundo Washabau (2004), podem evidenciar hipoproteinemia, devido à má nutrição, e leucocitose, associada à inflamação esofágica ou à pneumonia por aspiração. Achados esses que não foram vistos no resultado dos exames da paciente em questão. A leve eritropenia identificada pode não ser o valor real, pois uma anemia mais significativa pode ser mascarada pela desidratação, que nesse caso, pode não ter sido identificada clinicamente. A normoproteinemia também poderia ser devido à desidratação do animal, dissimulando uma possível hipoproteinemia (Lopes, 2007). A trombocitopenia demonstrada na análise hematológica da paciente pode ser explicada pela presença de uma quantidade discreta de agregados plaquetários (González, 2008). O recomendado seria repetir o exame hematológico, com uma coleta de sangue que não conferisse erro pré-analítico e após hidratação, para confirmação dos resultados (Lopes, 2007).

Para a terapêutica da pneumonia por aspiração, Washabau (2004) recomendou a realização de lavado broncoalveolar, cultura e teste de sensibilidade a antimicrobianos (TSA) para selecionar o antibiótico mais adequado para o(s) microrganismo(s) isolado(s). Longshore (2008) sugere a utilização de antibióticos de amplo espectro enquanto o resultado da cultura e TSA não são disponibilizados. No presente relato foi prescrito amoxicilina com clavulanato de potássio edcomo droga de escolha para o tratamento da pneumonia. A cultura e o TSA não foram realizados devido ao aumento dos custos para o tutor o que acabou inviabilizando tal conduta.

Segundo Willard (2015), até o presente momento, não há cura ou tratamento clínico que solucione a debilidade esofágica congênita. Indicase tratamento dietético conservador, a fim de evitar o agravamento da dilatação e a pneumonia por aspiração do conteúdo esofágico (Radlinsk, 2015). Classicamente, o animal é alimentado com alimentação pastosa, em uma plataforma elevada que requeira o animal em estação, com o apoio dos membros posteriores. Desta maneira, o esôfago cervical e torácico permanece em posição vertical no momento que o alimento é ingerido, permitindo o auxílio da gravidade na passagem do bolo alimentar do esôfago para o estômago (Langshore,
2008). É recomendando manter esta posição por cinco a dez minutos após a alimentação e ofertar várias refeições de aspecto pastoso ou sólido por dia em pequenas quantidades para evitar a retenção de alimento no esôfago. Essa abordagem vai de acordo com a terapêutica dietética aplicada no presente relato, mas sem sucesso terapêutico. Dos vários procedimentos terapêuticos preconizados, $\mathrm{o}$ tratamento cirúrgico representa uma opção de tratamento por proporcionar alívio dos sinais clínicos e melhora do estado nutricional (Torres, 2000). Devido o agravo dos episódios de regurgitação e perda de peso progressiva após duas semanas de tratamento dietético, foi indicado o tratamento cirúrgico pela técnica de cardioplastia esôfago diafragmático (Torres, 2000) que propõe reduzir artificialmente a paralisia da porção torácica caudal do esôfago e da cardia, através da atividade contrátil do diafragma durante os ciclos respiratórios (Torres, 2000). O único caso de insucesso relatado por Torres (2000) foi de um filhote de pastor alemão de três meses de idade. $\mathrm{O}$ mesmo foi operado em condições de extrema desnutrição e apresentava-se com pneumonia, entrando em óbito ao terceiro dia do pós-operatório devido às complicações respiratórias. $\mathrm{O}$ animal do presente relato apresentava-se caquético e com pneumonia que já estava sendo tratado. $\mathrm{O}$ prognóstico desses animais quando se apresentam caquéticos e com pneumonia é reservado já que o pós-operatório é mais complicado e necessita de uma maior participação do tutor (Torres, 2000). Quando possível, deve-se levar em consideração a possibilidade de condutas terapêuticas serem adotadas para melhorar o estado geral do paciente antes da cirurgia, pois isso melhora o prognóstico no pós-operatório. Torres (2000) relatou que alguns animais apresentaram uma diminuição progressiva do tamanho do esôfago após o procedimento, demonstrando à possibilidade do retorno a função normal do órgão. Esse dado não pode ser confirmado no presente trabalho, pois o animal faleceu antes que pudesse ter sido feito uma nova avaliação radiográfica. A hipótese que a cardioplastia esôfago diafragmática permite uma recuperação motora do esôfago, favorecendo a regeneração da inervação intrínseca da parede (Torres, 2000), baseia-se na imaturidade do centro motor esofágico do sistema nervoso central nos animais que desenvolvem megaesôfago congênito idiopático (Strombeck e Troya, 1976). Com o procedimento cirúrgico seria possível aumentar a sobrevida do filhote acometido, proporcionando 
tempo suficiente para que ocorra o completo desenvolvimento dos centros nervosos (Tan e Diamant, 1987). Apesar do sucesso obtido por Torres (2000), sugere-se que a técnica da cardioplastia esôfago diafragmática deve ser mais bem avaliada com relação aos possíveis benefícios que a mesma pode trazer para melhorar a qualidade de vida dos animais que desenvolvem megaesôfago generalizado.

Em seres humanos, Valezi et al. (2004) relataram a eficácia da cardiomiotomia com fundoplicatura parcial por vídeo-laparoscopia (Heller-Dor) no tratamento de 12 pacientes portadores de megaesôfago. Herbella et al. (1999), em estudo retrospectivo de 83 casos, de 1977 a 1995, também atestam que a cardiomiotomia associada à fundoplicatura é a cirurgia de escolha para o tratamento do megaesôfago em humanos, mostrando ser uma operação segura e com bons resultados. No entanto, como essa técnica ainda não foi realizada em cães com megaesôfago generalizado, ainda não existe informações sobre o seu benefício em animais. Apesar da existência de inúmeras técnicas para correção de disfunções esofágicas descritas na medicina humana, muitas dessas ainda não foram aplicadas a medicina veterinária, já que a maioria se baseia em resolver o problema de tensão localizado na região do esfíncter esofágico inferior, característico da acalasia humana e não do megaesôfago canino (Washabau, 2004).

O óbito do animal após 15 dias do procedimento cirúrgico e a não realização da necropsia torna difícil à definição da possível causa mortis do paciente em questão. Entretanto, algumas suposições podem ser colocadas, descartando ou diminuindo a possibilidade de algumas indagações. Por exemplo, o animal tinha pneumonia por aspiração leve, mas que estava sendo tratada e o mesmo não apresentava sinais clínicos importantes, não sendo, portanto, a possível causa de morte. Se o animal tivesse ido a óbito por causa de deiscência dos pontos, isso, provavelmente teria ocorrido com aproximadamente cinco dias, que é quando o processo de cicatrização deixa o tecido mais frágil a esse tipo de complicação. Contudo, o animal não foi acompanhado de forma ideal em um centro especializado para a realização da monitorização do paciente, aferição dos parâmetros vitais e manejo adequado de dor, nutrição e fluidoterapia, pois os tutores não tinham condição financeira. Isso torna o pós-operatório falho, já que o paciente não estava em condições ideais para ser cuidado em casa pelos tutores.

\section{Conclusão}

A caquexia e a pneumonia por aspiração são fatores que pioram o prognóstico, assim o procedimento cirúrgico deve ser realizado o mais precoce possível, antes do desenvolvimento destas complicações. Casos tais alterações já estejam presentes, tentar deixar o animal o mais estável possível antes do procedimento cirúrgico. Esses pacientes também podem se beneficiar da utilização de tubo gástrico para o fornecimento da alimentação adequada no pré e pós-operatório, possibilitando uma melhora rápida do animal. A técnica de cardioplastia esôfago diafragmática pode ser uma opção a ser considerada nos pacientes que não respondem ao tratamento conservador, entretanto é necessário selecionar adequadamente o animal que possa se beneficiar dessa técnica cirúrgica.

\section{Conflito de Interesse}

Os autores declaram não existir conflito de interesse.

\section{Referências}

Alves, N.M.; Silva, T.P.D.; Pereira, A.M.; Rosito, J.; Borges, M.M. Megaesôfago congênito em cão. Pubvet, 23(7): 1647, 2013.

Celano, R.M.G.; Neto, J.E.; TCBC-SP; Bottoni, A.; Gagliardi, D. TCBC-SP. Avaliação nutricional pré-operatória dos pacientes com megaesôfago não-avançado. Revista do Colégio Brasileiro de Cirurgiões, 34(3): 2531, 2007.

Forbes, D.C.; Leishman, D.E. Megaesophagus in cats. Canadian Veterinary Journal, 26(11): 354-356, 1985.

Gaiga, L.H.; Pigatto, J.A.T.; Brun, M.V. Megaesophagus and esophageal hiatal hernia complicating in dog: case report. Revista da FZVA, 13(2): 145-152, 2006.

Herbella, F.A.M.; Del Grande, J.C.; Lourenço, L.G.; Mansur, N.S.; Haddad, C.M. Resultados tardios da operação de Heller associada à fundoplicatura no tratamento do megaesôfago: análise de 83 casos. Revista da Associação Médica Brasileira, 45(4): 317322, 1999.

Longshore, R.C. Megaesôfago. In: Tilley, L.P.; Smith, F.W.K. Consulta veterinária em 5 
minutos: canina e felina. $3^{\mathrm{a}}$ ed. São Paulo: Manole, 2008. p.950-951.

Lopes, S.T.A.; Biondo, A.W.; Santos, A.P. Manual de patologia clínica veterinária. $3^{\mathrm{a}}$ ed. Santa Maria: UFSM, 2007. p. 48 - 53.

Radlinsk, M.G. Cirurgia do sistema digestório. In: Fossum, T.W. Cirurgia de pequenos animais. $4^{\mathrm{a}}$ ed. Rio de Janeiro: Elsevier, 2015. p. $424-456$.

Sturgess, C.P.; Dunn, J.K. Tratado de medicina de pequenos animais. São Paulo: Roca, 2001. 385-87p.

Strombeck, D.R.; Troya, L. Evaluation of lower motor neuron function on two dogs with megaesophagus. Journal of the American Veterinary Medical Association, 69(4): 411414, 1976.

Tan, B.J.K.; Diamant N.E. Assessment of the neural defect in a dog with idiopatic megaesophagus. Digestive Diseases and Sciences. New Series, 32(1): 76-80, 1987.

Tanaka, N.M.; Hoogevonink, N.; Tucholski, A. P.; Trapp, S. M.; Frehse, M. S. Megaesôfago em Cães. Revista Acadêmica: Ciências Agrárias e Ambientais, 8(3): 271-279, 2010.
Torres, P. Cardioplastía esófago-diafragmática como tratamiento del megaesófago total congénito idiopático en el perro. Archivos de Medicina Veterinária, 32(1): 46-52, 2000.

Washabau, R.J. Doenças do esôfago. In: Ettinger, S.J.; Feldman, E.C. Tratado de medicina interna veterinária: doenças do cão e gato. $5^{\mathrm{a}}$ ed. Rio de Janeiro: Guanabara Koogan, 2004. p.1205-1214.

Willard, M.D. Desordens da cavidade oral, faringe e esôfago. In: Couto, C.G.; Nelson, R.W. Medicina interna de pequenos animais. $5^{\mathrm{a}}$ ed. Rio de Janeiro: Elsevier, 2015. p.433-434.

Valezi, A.C.; TCBC-PR.; Junior, J.M.; Marson, A.C.; Brito, E.M.; ACBC- PR.; Souza, J.C.L. Tratamento do Megaesôfago chagásico grau II por laparoscopia: experiência em 12 casos. Revista do Colégio Brasileiro de Cirurgia, 31(3): 148-153, 2004.

Vega, C.B.; Carballo, S.F.; Ângulo, S.M.; De La Muela, M.S. Estudio de megaesófago congénito en perro study of congenital megaesophagus in dog. Revista Complutense de Ciencias Veterinarias, 3(2): 261-271, 2009. 